\title{
Analysis of Online Media Consumption during Initial Months of the COVID-19 Pandemic
}

\author{
Tarun $K$ Jella, $M P H^{1 *}$, Nicole Ducich $B S^{1}$, Taral $K$ Jella ${ }^{2}$, Mamta Singh, $M D, M S^{1,3}$, Johnie Rose, $M D$, \\ $P h D^{1,4}$, Heidi Gullett, MD, $M P H^{1,4}$ and Kurt Stange, MD, $P h D^{1,4}$
}

${ }^{1}$ School of Medicine, Case Western Reserve University, Cleveland, $\mathrm{OH}$, USA

${ }^{2}$ Emory College of Arts and Sciences, Emory University, Atlanta, GA, USA

${ }^{3}$ Division of General Medicine, VA Northeast Ohio Health System, Cleveland, OH, USA

${ }^{4}$ Center for Community Health Integration, Case Western Reserve University, Cleveland, OH, USA

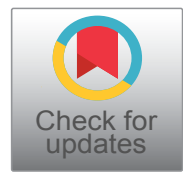

\begin{abstract}
As the COVID-19 cases continue to rise, individuals are seeking out online information regarding the pandemic at unprecedented rates. In volumes never seen before, and from various sources currently unknown to providers. This study may act as a guide for healthcare, public health, and government personnel to characterize the most popular destinations of COVID-19 related web searches, in order to dispel possible rumors and promote the consumption of credible information. We sought to characterize patterns in search engine utilization and website traffic related to the COVID-19 pandemic in relation to other large-scale disease outbreaks of the past two decades. Using the Google Trends and Similar Web analytics platforms, we found the relative popularity of the search term "Corona Virus" to more than double the peak search popularities for "Zika", "Ebola", and "Swine Flu". As of March 2020, 6.7\% of the total web traffic for "Corona Virus" was directed to the WHO and CDC, compared with $9.9 \%$ of web traffic directed to cable news networks, and $12.3 \%$ directed to daily newspapers. Increased scrutiny of these sites by health officials may be warranted to rapidly identify and counter the spread of false narratives regarding testing, treatment, or quarantine restrictions.
\end{abstract}

\section{Introduction}

Since December 2019, COVID-19 has caused a worldwide public health crisis resulting in over 1.3 million confirmed cases and almost 75,000 deaths [1]. As the affected population grows, there is a persistent need to identify and counteract unreliable sources of information. Recently, the WHO Director-General has prioritized correcting myths and rumors spread on various social media platforms [2]. It is crucial to understand where the public is seeking updates regarding the pandemic in order to dispel misinformation and promote the use of credible resources. This study analyzes patterns of online media consumption related to COVID-19 and compares contemporary web traffic to that of other recent major disease outbreaks.

\section{Methods}

We conducted an "infodemiological" [3] assessment of public search engine use and web traffic statistics. All data were collected using 'Google Trends'(GT) and the associated 'Similar Web'(SW) analytics tool, since globally, approximately $80-90 \%$ of internet users rely on Google [4]. Public search data is aggregated and indexed across millions of web pages to power both software platforms [5,6]. The former compares the usage of different keywords over time, while the latter compares traffic to 'competing' websites based on identical keyword searches [5,6].

GT data were extracted for the keywords "Zika," "Ebola," "Swine Flu," and "Corona Virus" from January 1, 2004 - March 31, 2020 (Figure 1). Data were reported as "Google Search Volume Index" (GSVI) values, representing the relative popularity of a term compared to all searches over a set timeframe. Next, the SW platform was used to analyze web traffic trends for "Corona Virus" search engine queries between January 2020 - March 2020 (Figure 2). All descriptive analyses were performed using R Studio Desktop (Version 1.2.5033).

\section{Results}

From January 2004 - March 2020, the peak GSVI value for

*Corresponding author: Dr. Tarun Jella, School of Medicine, Case Western Reserve University, 9501 Euclid Ave, Cleveland, OH 44106, USA Tel: (262)-422-0662

Accepted: November 28, 2020

Published online: November 30, 2020

Citation: Jella TK, Nicole DBS, Jella TK, et al. (2020) Analysis of Online Media Consumption during Initial Months of the COVID-19 Pandemic. Ann Public Health Reports 4(1):105-107 
Citation: Jella TK, Nicole DBS, Jella TK, et al. (2020) Analysis of Online Media Consumption during Initial Months of the COVID-19 Pandemic. Ann Public Health Reports 4(1):105-107

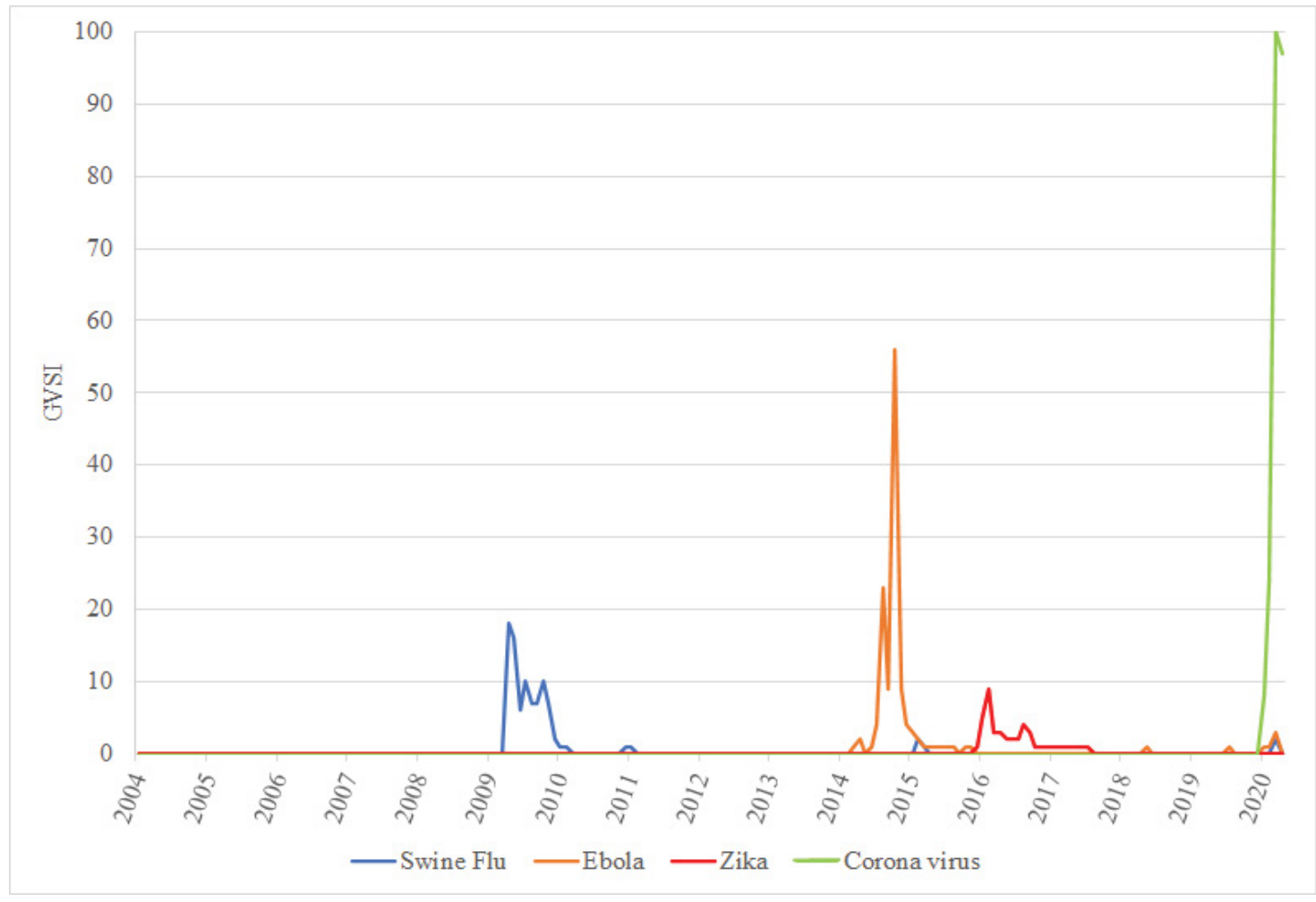

Figure 1: Comparison of Google Searches for Large-Scale Disease Outbreaks.

*Search data for each term are normalized against all searches and reported as google search volume index (GSVI) values ranging from 0 to 100 . A value of 50 indicates half the 'popularity' of a value of 100 , and vice versa.

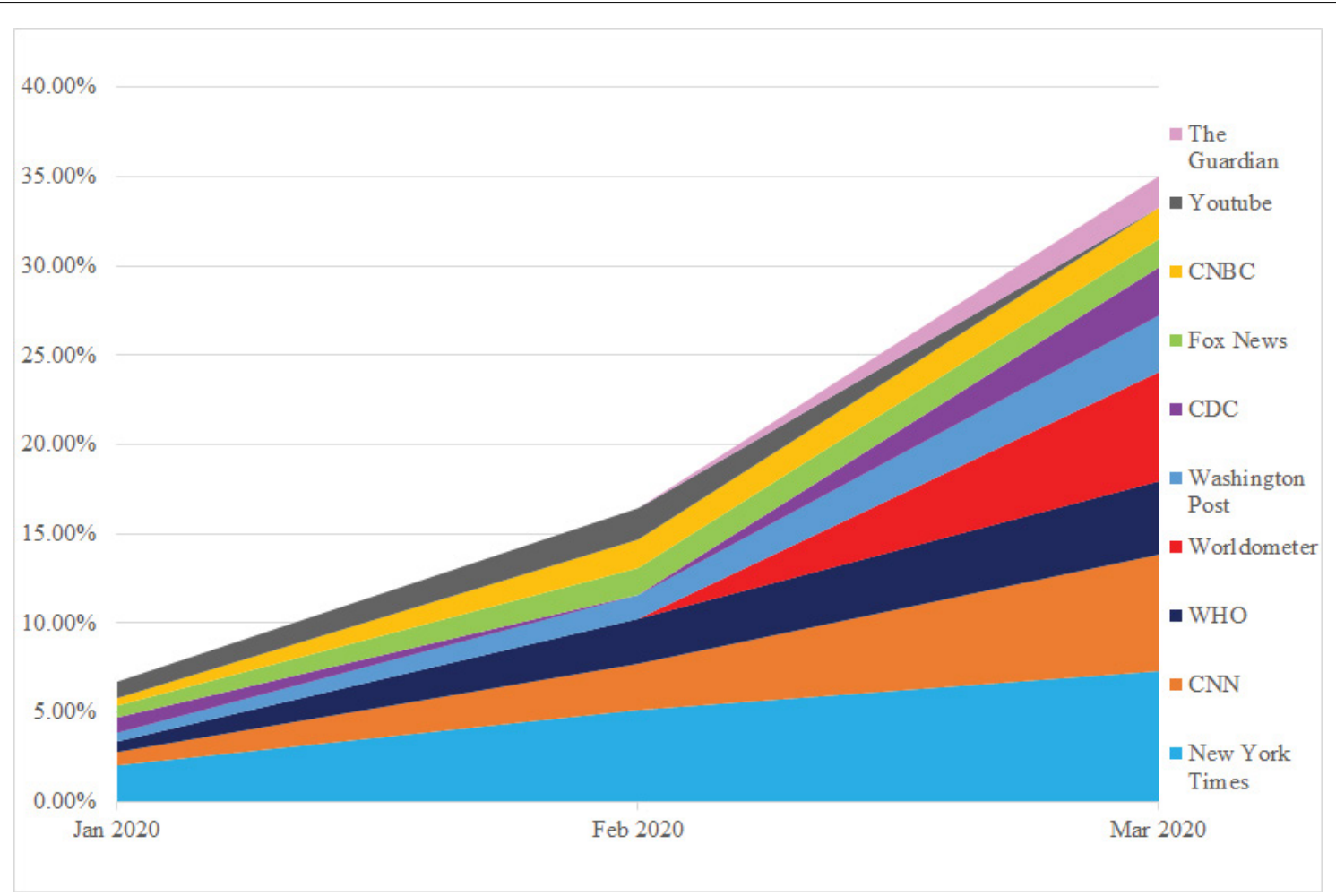

Figure 2: Web Traffic Trends of the Top 10 Destinations following "Corona Virus" Search Queries.

Similar web data January 2020 - March 2020: Source (pro.similarweb.com) 
the search term "Corona Virus" (100) was nearly double that of "Ebola" (56), more than five times that of "Swine Flu" (18), and over ten times that of "Zika" (9) (Figure 1).

The Center for Disease Control (CDC) and the World Health Organization (WHO) websites experienced among the highest traffic following "coronavirus" keyword searches (Figure 2). From January 2020 - March 2020, the monthly number of unique site visitors increased by $468.3 \%$ (32.48 million to 184.6 million) and $783.5 \%$ (20.69 million to 182.8 million) for the 'cdc.gov' and 'who.int' respectively. However, only $6.7 \%$ of the total web traffic for "coronavirus" was directed to these sites as of March 2020, compared with 9.9\% of web traffic directed to cable news network's (CNN, CNBC, and FOX), and $12.3 \%$ directed to daily newspapers, e.g. the New York Times and Washington Post.

\section{Discussion}

Our analysis documents the unprecedented scale of public interest in the COVID-19 pandemic with respect to search engine usage and online media consumption. The use of credible resources such as 'CDC.gov' and 'WHO.int' has increased dramatically since January, however, the vast majority of online information is still obtained from a diffuse mix of media websites. Increased scrutiny of these sites by health officials may be warranted to rapidly identify and counter the spread of false narratives regarding testing, treatment, or quarantine restrictions.

The greatest share of web traffic was concentrated amongst a small group of cable news network's and daily newspapers. Although these platforms strive for and likely achieve journalistic integrity, the potential harm stemming from any sensationalism which may arise is substantial. Non-conflicted public institutions such as the CDC and WHO which exist, in large part, to disseminate measured and consistent health information to guide individual and collective action should be trusted as a first line of defense against the COVID-19 "infodemic".

\section{Funding}

None.

\section{Conflicts of Interest}

None.

\section{References}

1. Dong E, Du H, Gardner L (2020) An interactive web-based dashboard to track COVID-19 in real time. Lancet Infect Dis 20:533534.

2. Ghebreyesus T A (2020) Munich security conference. World Health Organizations.

3. Eysenbach G (2009) Infodemiology and infoveillance: Framework for an emerging set of public health informatics methods to analyze search, communication and publication behavior on the internet. J Med Internet Res 11: e11.

4. Search Engine Market Worldwide (2019) Stat Counter Global Stats.

5. Stephens-Davidowitz S (2020) Google searches can help us find emerging Covid-19 outbreaks. The New York Times.

6. Koeze E, Popper N (2020) The virus changed the way we internet. The New York Times.

DOI: $10.36959 / 856 / 504$ 